\title{
Elevated SOX11 expression distinguishes basal-like human breast cancer.
}

Shahan Mamoor, MS ${ }^{1}$

$3 \quad{ }^{1}$ shahanmamoor@gmail.com

East Islip, NY 11730

Patients diagnosed with basal-like breast cancer face a more aggressive disease course and more dismal prognosis than patients diagnosed with luminal A and luminal B breast cancer molecular subtypes (1-4). We mined published microarray data $(5,6)$ to understand in an unbiased fashion the most distinguishing transcriptional features of tumors from patients with basal or basal-like subtype breast cancer. We observed transcriptome-wide differential expression of SRY-box 11, SOX11, when comparing tumors of patients with basal-like breast cancer with that of other PAM50 molecular subtypes. SOX11 mRNA was present at significantly higher quantities in the tumors of patients with basal-like breast cancer. Analysis of patient survival data revealed that SOX11 primary tumor expression was correlated with overall survival, with higher SOX11 associated with inferior outcomes - in basal-like patients but not in luminal A, luminal B, HER2+, or normal-like patients. Elevated SOX11 expression appears to distinguish basal-like human breast cancer from the other molecular subtypes.

Keywords: basal subtype, basal-like breast cancer, BLBC, systems biology of breast cancer, targeted therapeutics in breast cancer, breast cancer. 
Classification of disease types and disease subtypes enables development of enhanced diagnostic capabilities, prognostic information, as well as an understanding of human disease that can support superior medical, surgical and radiological treatment of complex diseases such as cancer. Molecular subtypes in human breast cancer include luminal A, luminal B, basal or basal-like, and HER2+. Systems-level analyses, such as comparative tumor transcriptome analyses, can inform such approaches. We mined published microarray data $(5,6)$ to identify the most significant transcriptional differences when comparing human breast cancer molecular subtypes. Integrative microarray dataset analysis has provided data illuminating genes whose expression define basal-like subtype breast cancers, and we present evidence here.

\section{Methods}

We utilized datasets GSE158309 (5) and GSE74667 (6) and for this global differential gene expression analysis of basal subtype human breast cancer in conjunction with GEO2R. GSE158308 was generated using Affymetrix Human Genome U133A Array technology with $n=52$ tumors from patients with basal-like breast breast and $n=410$ tumors from patients with luminal A, luminal B and HER2+ molecular subtypes; analysis was performed using platform GPL96. The data presented in the graph compare $n=52$ tumors from patients with basal-like breast cancer to $n=182$ tumors from patients with luminal B breast cancer. GSE74667 was generated using Agilent-014850 Whole Human Genome Microarray $4 \mathrm{x} 44 \mathrm{~K}$ G4112F technology with $n=23$ basal-like breast tumors and $n=72$ breast tumors of other molecular subtypes (luminal A, luminal B, HER2-enriched, and normal-like); analysis was performed using platform GPL6480. The Benjamini and Hochberg method of $p$-value adjustment was used for ranking of differential expression but raw $p$-values were used to assess statistical significance of global differential expression. Log-transformation of data was auto-detected, and the NCBI generated category of platform annotation was used. A statistical test was performed to evaluate whether SOX11 gene expression was significantly different between the tumors of patients with basal and non-basal molecular subtype breast cancer (luminal B) using a two-tailed t-test with Welch's correction. For Kaplan-Meier survival analysis, we used the Kaplan-Meier plotter tool (8) for correlation of SOX11 mRNA expression levels with overall survival in $n=1879$ breast cancer patients, $n=431$ basal subtype breast cancer patients, $n=596$ luminal A subtype breast cancer patients, $n=439$ luminal B subtype breast cancer patients, $n=362$ HER2 + subtype breast cancer patients, and $n=51$ normal-like subtype breast cancer patients. .

\section{Results}

SOX11 is among the genes whose expression is most different when comparing basal-like breast cancers with breast cancers of other molecular subtypes.

We used published microarray data (5) to compare the whole tumor transcriptomes of 52 patients with basal subtype breast cancer and 410 patients with breast cancer of other molecular subtypes (luminal A, luminal B and HER2+) to identify genes whose expression marked the basal-like molecular subtype. We observed transcriptome-wide differential expression of SRY-box 11, SOX11, when comparing the tumors of patients with basal-like cancer with that of patients with luminal A, luminal B and HER2+ cancers (Table 1). When sorting each of the transcripts whose expression was measured globally, SOX11 expression ranked 476 out of 22283 total transcripts, equating to $97.9 \%$ differential expression; this was statistically significant ( $p$-value: 4.37E-05).

Analysis of a second published microarray dataset, comparing tumor transcriptome data from a separate cohort of patients 23 patients with basal-like breast cancer and 72 patients with breast cancer of other molecular subtype (luminal A, luminal B, HER2-enriched, and normal-like) (6) again revealed 
transcriptome-wide differential expression of SOX11 in patients with basal subtype human breast cancer (Table 2). When sorting each of the transcripts whose expression was measured globally, SOX11 expression ranked 336 out of 31157 total transcripts, equating to $98.9 \%$ differential expression; this was statistically significant ( $p$-value: 4.61E-08). Thus, the data suggested differential expression of SOX11 was a fundamental feature of basal-like breast cancer in humans rather than an artifact of a single microarray dataset or research group, or a finding derived from cell culture or an animal model that was not relevant to human breast cancer.

$\underline{\text { SOX11 expression correlates with overall survival in basal subtype breast cancer patients. }}$

We asked whether primary tumor expression of SOX11 was correlated with survival outcomes in patients with basal-like breast cancer. We observed a correlation between primary tumor expression of SOX11 and overall survival (OS) in patients with basal-like breast cancer which approached statistical significance (Figure 2; $p$-value: 0.06 ; hazard ratio: 1.44 (0.98-2.12)). SOX11 primary tumor expression was a negative prognostic indicator in patients with basal-like breast cancer. Patients with low tumor expression of SOX11 possessed median OS of 90 months, while patients with high tumor expression of SOX11 possessed median OS of 57.8 months (Table 3 ).

We also observed a statistically significant correlation between primary tumor expression of SOX11 and overall survival (OS) in patients with breast cancer of any (all) molecular subtype(s) (Figure 2; $p$-value: 2.9 e- 05 ; hazard ratio: 1.49 (1.24-1.8)). SOX11 primary tumor expression was a negative prognostic indicator with respect to overall survival in patients with breast cancer. Patients with low tumor expression of SOX11 possessed median OS of 125.92 months, while patients with high tumor expression of SOX11 possessed median OS of 69.73 months (Table 4).

SOX11 primary tumor expression was not correlated with overall survival in luminal A subtype breast cancer (Figure 2; $p$-value: 0.83; hazard ratio: 1.05 (0.69-1.6)), in luminal B subtype breast cancer (Figure 2; $p$-value: 0.38; hazard ratio: 1.17 (0.82-1.66), in HER2+ breast cancer (Figure 2; $p$-value: 0.25; hazard ratio: $0.8(0.54-1.18)$ ), or in normal-like breast cancer (Figure 2; $p$-value: 0.76 ; hazard ratio: 0.86 $(0.32-2.3))$.

\section{Discussion}

We find here that SOX11 is among the genes whose expression is most different when comparing basal-like breast cancers (BLBC) to cancers of other molecular subtypes, with significantly more SOX11 mRNA in BLBC. Kaplan-Meier analysis of patient survival data revealed that SOX11 expression was correlated with overall survival in human patients with basal-like breast cancer, with higher expression of SOX11 associated with inferior outcomes. SOX11 is structurally related to SOX2 which functions in pluripotency in embryonic stem cells (8) and as a "pioneer" transcription factor (9). SOX11 may be one factor that specifies tumor subtype identity in basal-like breast cancer. 


\section{References}

1. Rakha, E.A., Reis-Filho, J.S. and Ellis, I.O., 2008. Basal-like breast cancer: a critical review. Journal of clinical oncology, 26(15), pp.2568-2581.

2. Milioli, H.H., Tishchenko, I., Riveros, C., Berretta, R. and Moscato, P., 2017. Basal-like breast cancer: molecular profiles, clinical features and survival outcomes. BMC medical genomics, 10(1), pp.1-17.

3. Foulkes, W.D., Smith, I.E. and Reis-Filho, J.S., 2010. Triple-negative breast cancer. New England journal of medicine, 363(20), pp.1938-1948.

4. Dietze, E.C., Sistrunk, C., Miranda-Carboni, G., O'regan, R. and Seewaldt, V.L., 2015. Triple-negative breast cancer in African-American women: disparities versus biology. Nature Reviews Cancer, 15(4), pp.248-254.

5. Heimes, A.S., Härtner, F., Almstedt, K., Krajnak, S., Lebrecht, A., Battista, M.J., Edlund, K., Brenner, W., Hasenburg, A., Sahin, U. and Gehrmann, M., 2020. Prognostic significance of interferon- $\gamma$ and its signaling pathway in early breast cancer depends on the molecular subtypes. International Journal of Molecular Sciences, 21(19), p.7178

6. She, Q.B., Gruvberger-Saal, S.K., Maurer, M., Chen, Y., Jumppanen, M., Su, T., Dendy, M., Lau, Y.K.I., Memeo, L., Horlings, H.M. and van de Vijver, M.J., 2016. Integrated molecular pathway analysis informs a synergistic combination therapy targeting PTEN/PI3K and EGFR pathways for basal-like breast cancer. BMC cancer, 16(1), pp.1-16.

7. Györffy, B., Lanczky, A., Eklund, A.C., Denkert, C., Budczies, J., Li, Q. and Szallasi, Z., 2010. An online survival analysis tool to rapidly assess the effect of 22,277 genes on breast cancer prognosis using microarray data of 1,809 patients. Breast cancer research and treatment, 123(3), pp.725-731.

8. Takahashi, K. and Yamanaka, S., 2006. Induction of pluripotent stem cells from mouse embryonic and adult fibroblast cultures by defined factors. Cell, 126(4), pp.663-676.

9. Dodonova, S.O., Zhu, F., Dienemann, C., Taipale, J. and Cramer, P., 2020. Nucleosome-bound SOX2 and SOX11 structures elucidate pioneer factor function. Nature, 580(7805), pp.669-672. 


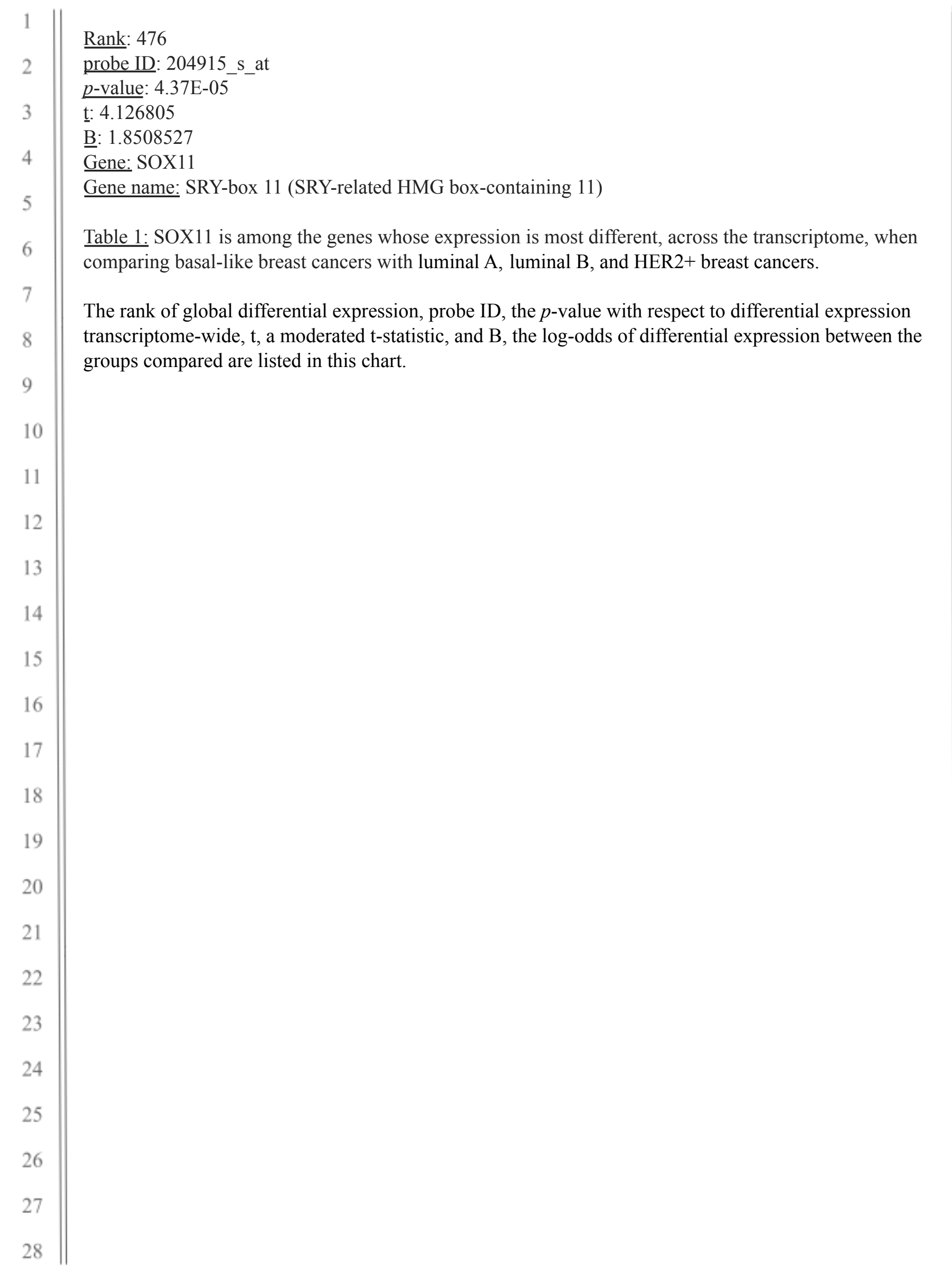




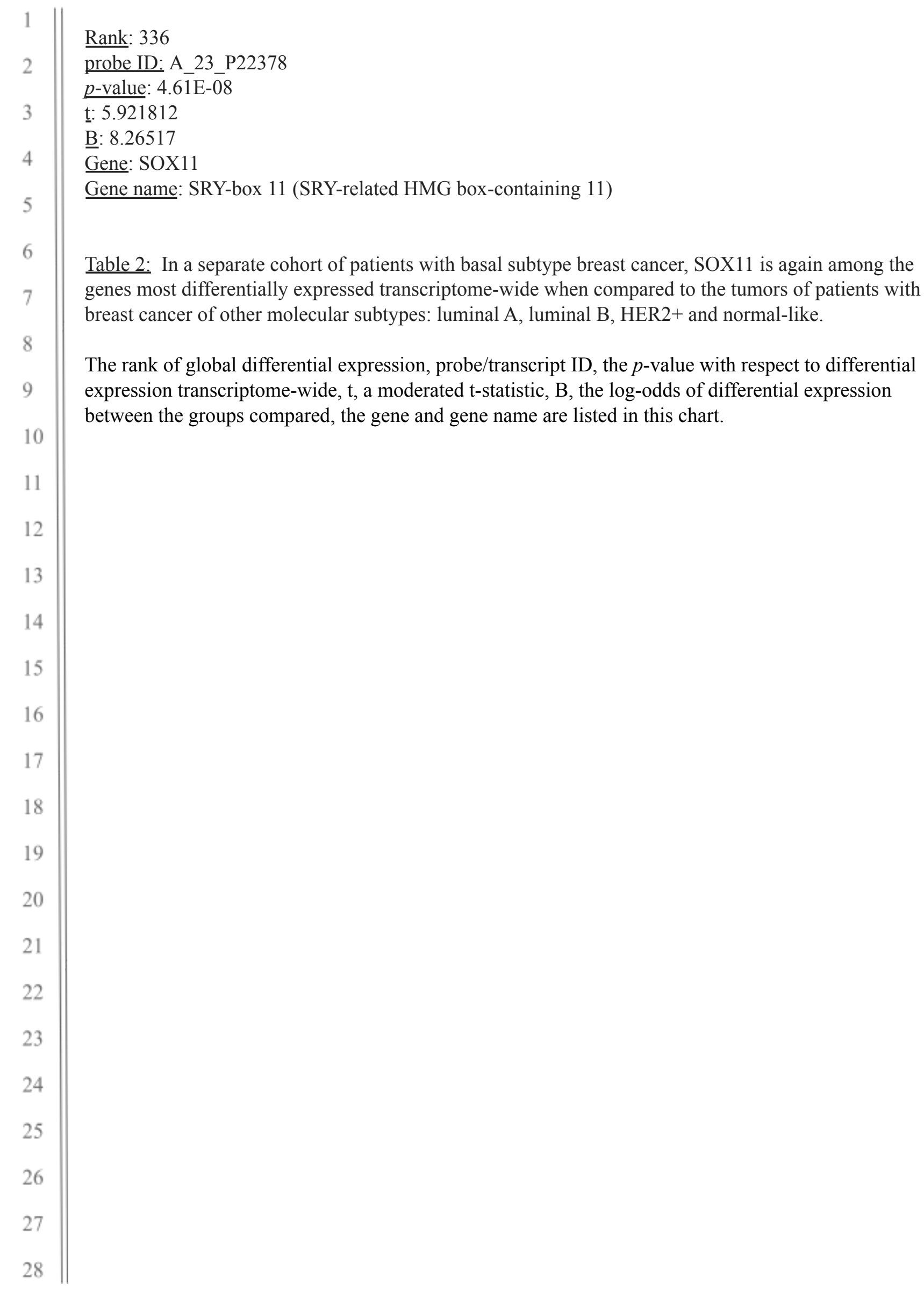




\section{sox11} line depicting the mean.
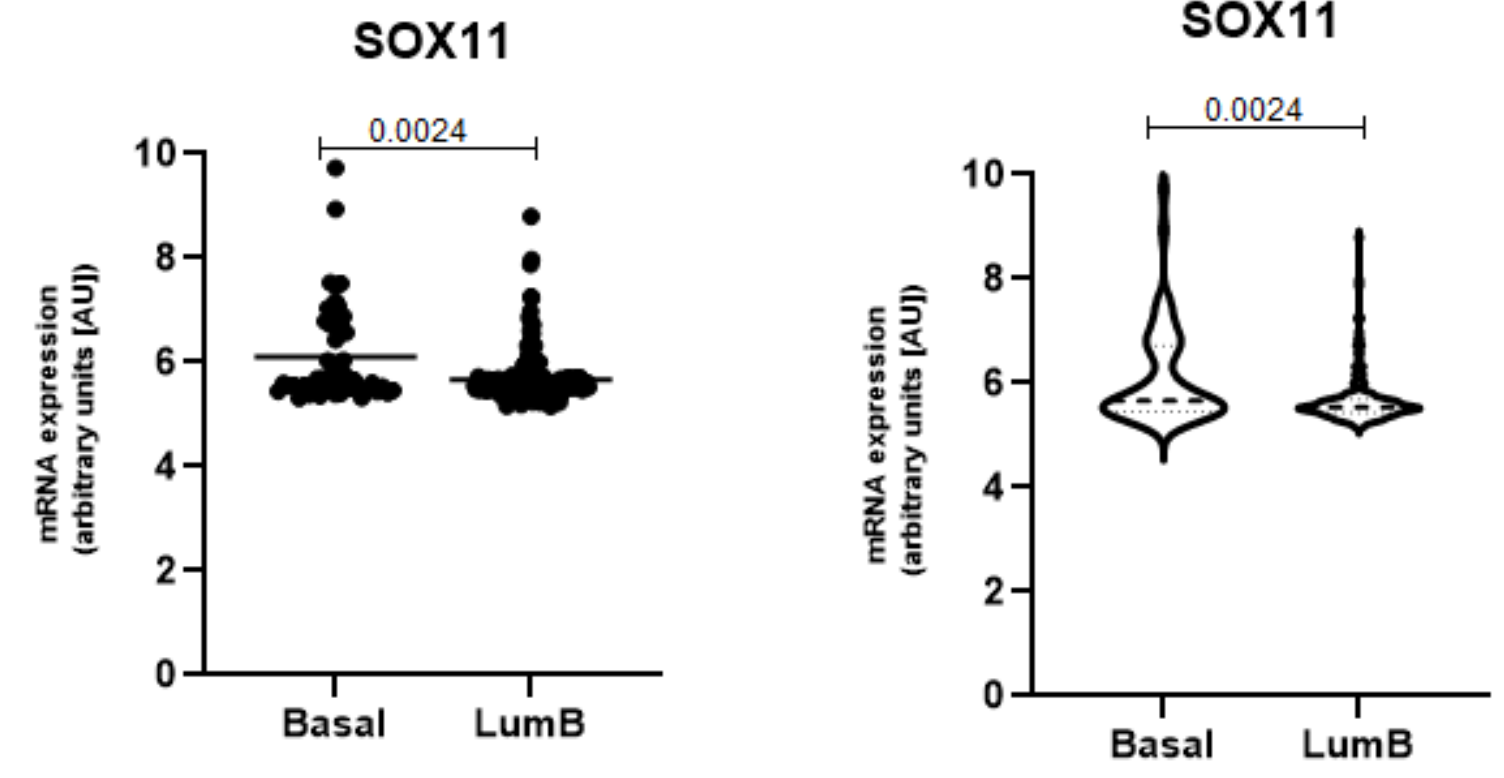

Figure 1: SOX11 expression is significantly higher in basal-like breast cancers.

Depicted is mRNA expression of SOX11 in tumors from patients with basal-like breast cancer and from patients with cancer of another molecular subtype, luminal B. On the left, the horizontal bar corresponds to the mean value, and the result of a statistical test evaluating the significance of difference in expression between the two groups compared, a $p$-value, is listed above. On the right, the same data is presented, but in a violin plot, with the two minor dotted lines depicting the 25 th and 75 th percentile and the major dotted 


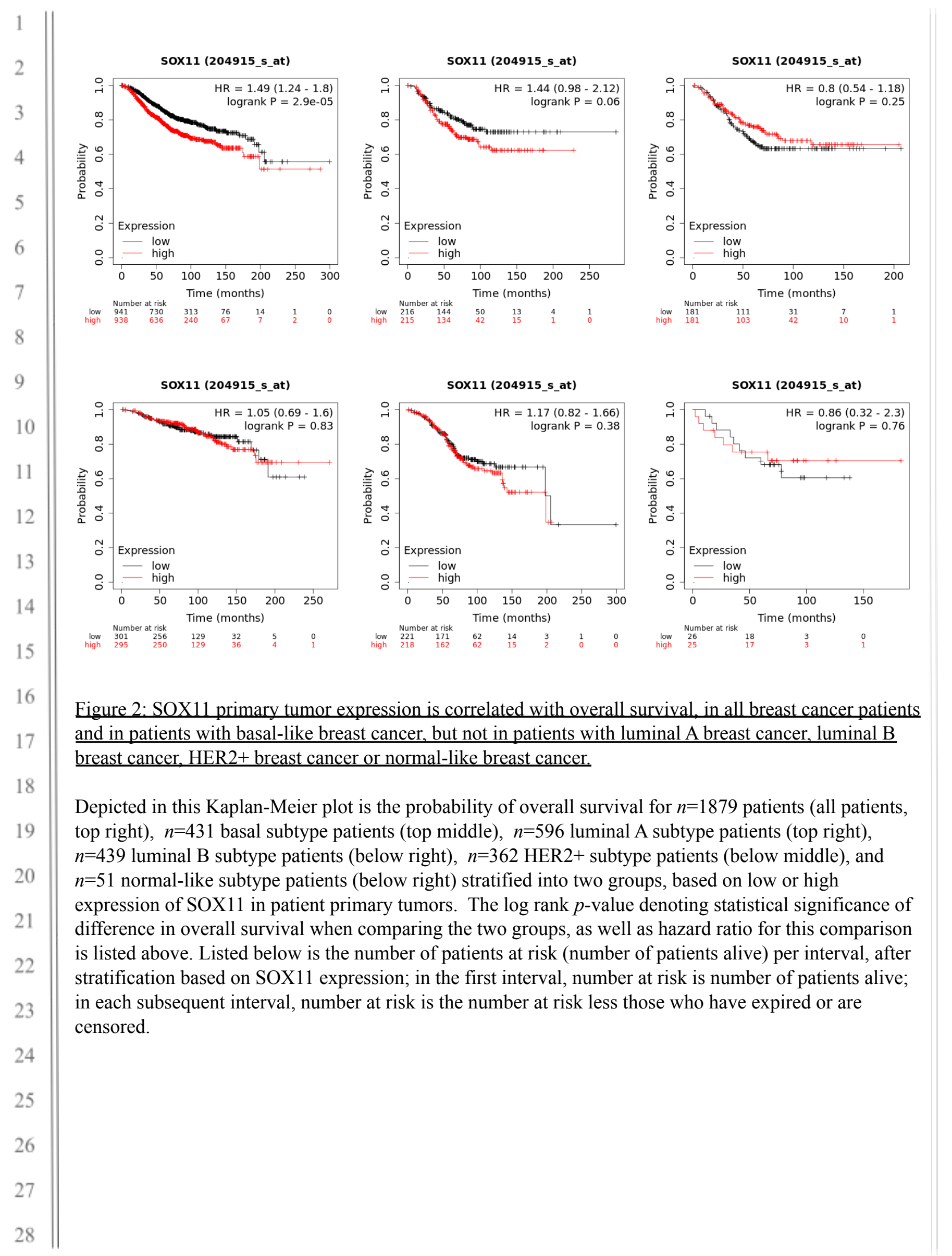




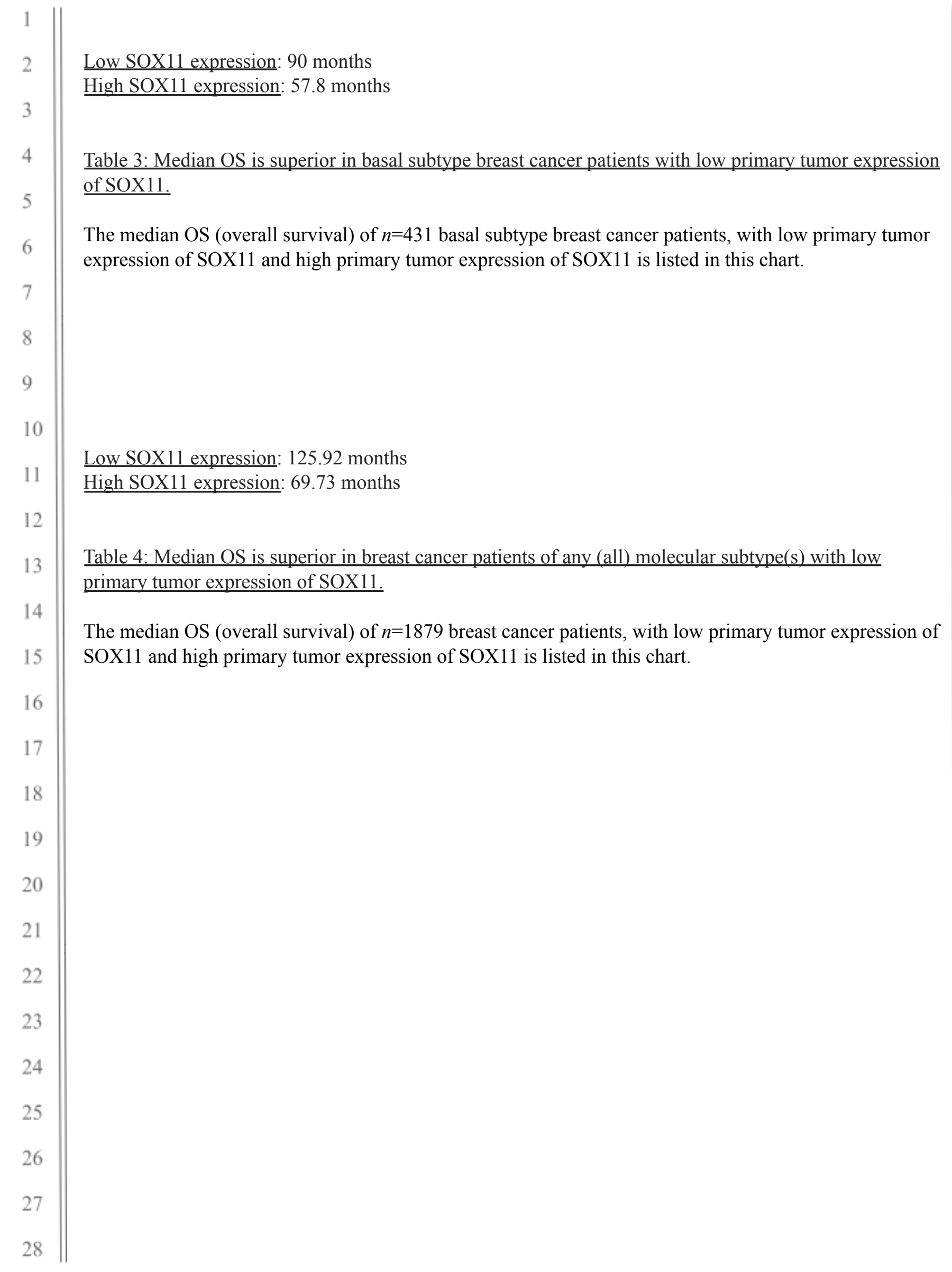

